\title{
Nonlinear Adaptive Control of a Chemical Reactor
}

\author{
PETR DOSTÁL, FRANTIŠEK GAZDOŠ, VLADIMÍR BOBÁL, *MONIKA BAKOŠOVÁ \\ Tomas Bata University in Zlin \\ Department of Process Control \\ Centre of Polymer Systems \\ nam. T.G. Masaryka 5555, 76001 Zlin \\ CZECH REPUBLIC \\ \{dostalp, gazdos, bobal\}@fai.utb.cz \\ *Slovak University of Technology \\ Faculty of Chemical and Food Technology \\ Radlinskeho 9, 81237 Bratislava \\ SLOVAK REPUBLIC \\ monika.bakosova@stuba.sk
}

\begin{abstract}
The paper deals with continuous-time nonlinear adaptive control of a continuous stirred tank reactor. The control strategy is based on an application of the controller consisting of a linear and nonlinear part. The static nonlinear part is derived in the way of an inversion and exponential approximation of measured or simulated input-output data. The design of the dynamic linear part is based on approximation of nonlinear elements in the control loop by a continuous-time external linear model with directly estimated parameters. In the control design procedure, the polynomial approach with the pole assignment method is used. The nonlinear adaptive control is tested by simulations on the nonlinear model of the CSTR with a consecutive exothermic reaction.
\end{abstract}

Key-Words: - Chemical reactor, nonlinear control, adaptive control, polynomial method, pole assignment.

\section{Introduction}

From the system theory point of view, continuous stirred tank reactors (CSTRs) belong to a class of nonlinear systems with mathematical models described by sets of nonlinear differential equations. Various models of CSTRs can be found in e.g. [4], [10] and [12].

It is well known that the control of chemical reactors often represents very complex problem. The control problems are due to the process nonlinearity, high input-output sensitivity as well as to other unpleasant behaviour. The process with such properties is hardly controllable by conventional control methods, and, its effective control requires application some of advanced methods.

One possible method to cope with this problem exploits a linear adaptive controller with parameters computed and readjusted on the basis of recursively estimated parameters of an appropriate chosen continuous-time external linear model (CT ELM) of the process. Some results obtained by this method can be found in e.g. [5] and [6].

An effective approach to the control of CSTRs and similar processes utilizes various methods of the nonlinear control (NC). Several modifications of the
NC theory are described in e.g. [1], [8] or [15].

Especially, a part of the NC methods exploits factorization of nonlinear models of the plants on linear and nonlinear parts, e.g. [2], [3], [13], [14] or[16].

In this paper, the CSTR control strategy is based on an application of the controller consisting of a static nonlinear part (SNP) and dynamic linear part (DLP). The static nonlinear part is obtained from simulated or measured steady-state characteristic of the CSTR, its inversion, exponential approximation, and, subsequently, its differentiation. On behalf of development of the linear part, the SNP including the nonlinear model of the CSTR is approximated by a CT external linear model. For the CT ELM parameter estimation, the direct estimation in terms of filtered variables is used, see e.g. [7] and[11]. Then, the resulting CT controller is derived using the polynomial approach and pole assignment method, e.g. [9]. The simulations are performed on a nonlinear model of the CSTR with a consecutive exothermic reaction.

\section{Model of the CSTR}

Consider a CSTR with the first order consecutive 
exothermic reaction according to the scheme $\mathrm{A} \stackrel{k_{1}}{\longrightarrow} \mathrm{B} \stackrel{k_{2}}{\longrightarrow} \mathrm{C}$ and with a perfectly mixed cooling jacket. Using the usual simplifications, the model of the CSTR is described by four nonlinear differential equations

$$
\begin{gathered}
\frac{d c_{\mathrm{A}}}{d t}=-\left(\frac{q_{\mathrm{r}}}{V_{\mathrm{r}}}+k_{1}\right) c_{\mathrm{A}}+\frac{q_{\mathrm{r}}}{V_{\mathrm{r}}} c_{\mathrm{Af}} \\
\frac{d c_{\mathrm{B}}}{d t}=-\left(\frac{q_{\mathrm{r}}}{V_{\mathrm{r}}}+k_{2}\right) c_{\mathrm{B}}+k_{1} c_{\mathrm{A}}+\frac{q_{\mathrm{r}}}{V_{\mathrm{r}}} c_{\mathrm{Bf}} \\
\frac{d T_{\mathrm{r}}}{d t}=\frac{h_{\mathrm{r}}}{\left(\rho c_{p}\right)_{\mathrm{r}}}+\frac{q_{\mathrm{r}}}{V_{\mathrm{r}}}\left(T_{\mathrm{rf}}-T_{\mathrm{r}}\right)+\frac{A_{\mathrm{h}} U}{V_{\mathrm{r}}\left(\rho c_{p}\right)_{\mathrm{r}}}\left(T_{\mathrm{c}}-T_{\mathrm{r}}\right) \\
\frac{d T_{\mathrm{c}}}{d t}=\frac{q_{\mathrm{c}}}{V_{\mathrm{c}}}\left(T_{c \mathrm{f}}-T_{\mathrm{c}}\right)+\frac{A_{\mathrm{h}} U}{V_{\mathrm{c}}\left(\rho c_{p}\right)_{\mathrm{c}}}\left(T_{\mathrm{r}}-T_{\mathrm{c}}\right)
\end{gathered}
$$

with initial conditions $c_{\mathrm{A}}(0)=c_{\mathrm{A}}^{\mathrm{s}}, \quad c_{\mathrm{B}}(0)=c_{\mathrm{B}}^{\mathrm{s}}$, $T_{\mathrm{r}}(0)=T_{\mathrm{r}}^{\mathrm{s}}$ and $T_{\mathrm{c}}(0)=T_{\mathrm{c}}^{\mathrm{s}}$. Here, $t$ is the time, $c$ are concentrations, $T$ are temperatures, $V$ are volumes, $\rho$ are densities, $c_{p}$ are specific heat capacities, $q$ are volumetric flow rates, $A_{\mathrm{h}}$ is the heat exchange surface area and $U$ is the heat transfer coefficient. The subscripts are denoted ( $)_{\mathrm{r}}$ for the reactant mixture, ()$_{c}$ for the coolant, ()$_{f}$ for steady-state inputs and the superscript ( $)^{\mathrm{s}}$ for initial conditions. The reaction rates and the reaction heat are expressed as

$$
\begin{gathered}
k_{j}=k_{0 j} \exp \left(\frac{-E_{j}}{R T_{\mathrm{r}}}\right), j=1,2 \\
h_{\mathrm{r}}=h_{1} k_{1} c_{\mathrm{A}}+h_{2} k_{2} c_{\mathrm{B}}
\end{gathered}
$$

where $k_{0}$ are pre-exponential factors, $E$ are activation energies and $h$ are reaction entalpies. The values of all parameters, inlet values and steadystate values with used units are given in Table. 1 .

Table 1. Parameters, steady-state inputs and initial conditions.

\begin{tabular}{|ll|}
\hline$V_{\mathrm{r}}=1.2 \mathrm{~m}^{3}$ & $c_{p \mathrm{r}}=4.05 \mathrm{~kJ} \mathrm{~kg}^{-1} \mathrm{~K}^{-1}$ \\
$V_{\mathrm{c}}=0.64 \mathrm{~m}^{3}$ & $c_{p \mathrm{c}}=4.18 \mathrm{~kJ} \mathrm{~kg}^{-1} \mathrm{~K}^{-1}$ \\
$\rho_{\mathrm{r}}=985 \mathrm{~kg} \mathrm{~m}^{-3}$ & $A_{\mathrm{h}}=5.5 \mathrm{~m}^{2}$ \\
$\rho_{\mathrm{c}}=998 \mathrm{~kg} \mathrm{~m}^{-3}$ & $U=43.5 \mathrm{~kJ} \mathrm{~m}^{-2} \mathrm{~min}^{-1} \mathrm{~K}^{-1}$ \\
$k_{10}=5.616 \times 10^{16} \mathrm{~min}^{-1}$ & $E_{1} / R=13477 \mathrm{~K}$ \\
$k_{20}=1.128 \times 10^{18} \mathrm{~min}^{-1}$ & $E_{2} / R=15290 \mathrm{~K}$ \\
$h_{1}=4.8 \times 10^{4} \mathrm{~kJ} \mathrm{kmol}^{-1}$ & $h_{2}=2.2 \times 10^{4} \mathrm{~kJ} \mathrm{kmol}^{-1}$ \\
\hline$c_{\mathrm{A}}^{\mathrm{s}}=1.5796 \mathrm{kmol} \mathrm{m}^{-3}$ & $c_{\mathrm{B}}^{\mathrm{s}}=1.1975 \mathrm{kmol} \mathrm{m}^{-3}$ \\
$T_{\mathrm{r}}^{\mathrm{s}}=324.80 \mathrm{~K}$ & $T_{\mathrm{c}}^{\mathrm{s}}=306.28 \mathrm{~K}$ \\
\hline$c_{\mathrm{Af}}^{\mathrm{s}}=2.85 \mathrm{kmol} \mathrm{m}^{-3}$ & $c_{\mathrm{Bf}}^{\mathrm{s}}=0 \mathrm{kmol} \mathrm{m}^{-3}$ \\
$T_{\mathrm{rf}}^{\mathrm{s}}=323 \mathrm{~K}$ & $T_{\mathrm{cf}}^{\mathrm{s}}=293 \mathrm{~K}^{\mathrm{s}}$ \\
$q_{\mathrm{r}}^{\mathrm{s}}=0.08 \mathrm{~m}^{3} \mathrm{~min}^{-1}$ & $q_{\mathrm{c}}^{\mathrm{s}}=0.08 \mathrm{~m}^{3} \mathrm{~min}^{-1}$ \\
\hline
\end{tabular}

In term of the practice, only the coolant flow rate can be taken into account as the control input. As the controlled output, the reactant temperature is considered. For the control purposes, the control input $u(t)$ and the controlled output $y(t)$ are defined as deviations from steady values

$$
u(t)=q_{\mathrm{c}}(t)-q_{\mathrm{c}}^{\mathrm{s}}, \quad y(t)=T_{\mathrm{r}}(t)-T_{\mathrm{r}}^{\mathrm{s}}
$$

The dependence of the reactant temperature on the coolant flow rate in the steady-state is in Fig. 1.

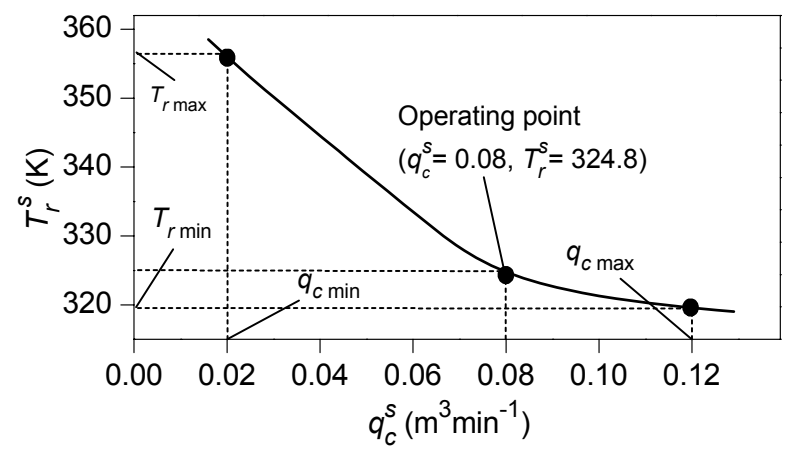

Fig. 1. Dependence of the reactant temperature on the coolant flow rate in the steady-state.

In subsequent control simulations, the operating interval for $q_{c}$ has been determined as

$$
q_{\mathrm{c} \min } \leq q_{\mathrm{c}}(t) \leq q_{\mathrm{c} \max }
$$

With regard to the purposes of a latter steadystate characteristic approximation, the values $q_{\mathrm{cL}}$ and $q_{\mathrm{cU}}$ are established that denote the lower and upper bound of $q_{\mathrm{c}}^{\mathrm{s}}$ used for the approximation, and, $T_{\mathrm{rU}}$ and $T_{\mathrm{rL}}$ to them corresponding temperatures.

\section{Controller Design}

As previously introduced, the controller consist of a static nonlinear part and a dynamic linear part as shown in Fig. 2.

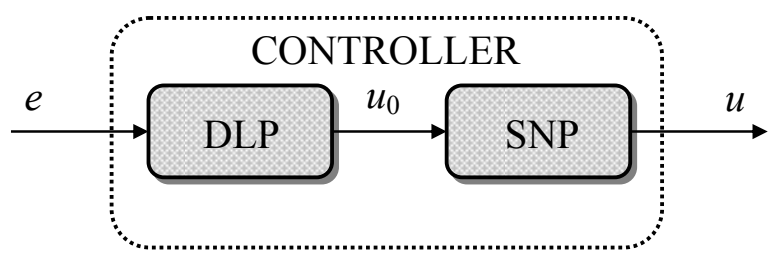

Fig. 2. The controller scheme.

The DLP creates a linear dynamic relation betveen the tracking error $e(t)$ and $u_{0}(t)=\Delta T_{\mathrm{rw}}(t)$ which represents a difference of the reactant temperature adequate to its desired value. Then, the SNP generates a static nonlinear relation betveen $u_{0}$ and a 
corresponding increment (decrement) of the coolant flow rate.

\subsection{Nonlinear part of the controller}

The SNP derivation appears from a simulated or measured steady-state charasteristic. The coordinates on the graph axis are defined as

$$
\gamma=\frac{q_{\mathrm{c}}^{\mathrm{s}}-q_{\mathrm{cL}}}{q_{\mathrm{cL}}}, \psi=T_{\mathrm{r}}^{\mathrm{s}}-T_{\mathrm{rL}}
$$

where $q_{\mathrm{cL}}$ is the lower bound in the interval

$$
q_{\mathrm{cL}} \leq q_{\mathrm{c}}^{\mathrm{s}} \leq q_{\mathrm{cU}}
$$

and, $T_{\mathrm{rL}}$ is the temperature corresponding to $q_{\mathrm{cU}}$.

It can be recommended to select the interval (10) slightly longer than (8). In this paper, lower and upper values in (8) and (10) were chosen $q_{\mathrm{cL}}=0.016, \quad q_{\mathrm{c} \min }=0.02, \quad q_{\mathrm{c} \max }=0.12, \quad$ and $q_{\mathrm{cU}}=0.13$.

In term of the practice, it can be supposed that the measured data will be affected by measurement errors. The simulated steady-state characteristic that corresponds to reality is shown in Fig. 3.

Making the replacement of coordinates, the inverse of this characteristic can be approximated by a function from the ring of polynomial, exponential, rational, eventually, by



Fig. 3. Simulated characteristics $\psi=f(\gamma)$.

other type functions. Here, the second order exponential approximate function has been found in the form

$$
\begin{aligned}
\gamma= & -74071.7+2.4589 \exp \left(-\frac{\psi}{3.967}\right)+ \\
& +74076 \exp \left(-\frac{\psi}{697475}\right)
\end{aligned}
$$

The inverse characteristic together with its approximation is in Fig. 4.

Now, a difference of the coolant flow rate $u(t)=\Delta q_{\mathrm{c}}(t)$ in the output of the SNP can be



Fig. 4. Simulated and approximated inverse relation $\gamma=\varphi(\psi)$.

computed for each $T_{\mathrm{r}}$ as

$$
u(t)=\Delta q_{\mathrm{c}}(t)=q_{\mathrm{cL}}\left(\frac{d \gamma}{d \psi}\right)_{\psi\left(T_{\mathrm{r}}\right)} u_{0}(t)
$$

The derivative of the approximate function (11) shown in Fig. 5 takes the form

$$
\begin{aligned}
\frac{d \gamma}{d \psi} & =-0.6198 \exp \left(-\frac{\psi}{3.967}\right)- \\
& -0.1062 \exp \left(-\frac{\psi}{697475}\right)
\end{aligned}
$$



Fig. 5. Derivative of $\gamma$ with respect to $\psi$.

\subsection{CT external linear model of nonlinear elements}

The second order CT ELM of the SNP in conjuction with a nonlinear model of the CSTR was chosen on the basis of pre-computed step responses of the $\mathrm{SNP}+\mathrm{CSTR}$ in the form of the second order linear differential equation

$$
\ddot{y}(t)+a_{1} \dot{y}(t)+a_{0} y(t)=b_{0} u_{0}(t)
$$

or, in the transfer function representation as

$$
G(s)=\frac{Y(s)}{U_{0}(s)}=\frac{b(s)}{a(s)}=\frac{b_{0}}{s^{2}+a_{1} s+a_{0}}
$$

where $s$ is the complex variable (parameter of the Laplace transform). 


\subsection{CT ELM parameter estimation}

The method of the CT ELM parameter estimation can be briefly carried out as follows.

Since the derivatives of both input and output cannot be directly measured, filtered variables $u_{0 \mathrm{f}}$ and $y_{\mathrm{f}}$ are established as the outputs of filters

$$
\begin{aligned}
c(\sigma) u_{0 \mathrm{f}}(t) & =u_{0}(t) \\
c(\sigma) y_{\mathrm{f}}(t) & =y(t)
\end{aligned}
$$

where $\sigma=d / d t$ is the derivative operator, $c(\sigma)$ is a stable polynomial in $\sigma$ that fulfills the condition $\operatorname{deg} c(\sigma) \geq \operatorname{deg} a(\sigma)$. It can be easily proved that the transfer behavior among filtered and among nonfiltered variables are equivalent. Using the $L$ transform of (15) and (16), the expressions

$$
\begin{gathered}
c(s) U_{0 \mathrm{f}}(s)=U_{0}(s)+\mu_{1}(s) \\
c(s) Y_{\mathrm{f}}(s)=Y(s)+\mu_{2}(s)
\end{gathered}
$$

can be obtained with $\mu_{1}$ and $\mu_{2}$ as polynomials of initial conditions. Substituting (17) and (18) into (14), and, after some manipulations, the relation between transforms of the filtered input and output takes the form

$$
\begin{aligned}
Y_{\mathrm{f}}(s)= & \frac{b(s)}{a(s)} U_{0 \mathrm{f}}(s)+M(s)= \\
& =G(s) U_{0 \mathrm{f}}(s)+M(s)
\end{aligned}
$$

where $M(s)$ is a rational function as the transform of any function $\mu(t)$ which expresses an influence of initial conditions of filtered variables.

The filtered variables including their derivatives can be sampled from filters (15) and (16) in discrete time intervals $t_{k}=k T_{\mathrm{S}}, k=0,1,2, \ldots \quad$ where $T_{\mathrm{S}}$ is the sampling period. Denoting $\operatorname{deg} a=n$ and $\operatorname{deg} b=$ $m$, the regression vector is defined as

$$
\begin{array}{r}
\boldsymbol{\Phi}\left(t_{k}\right)=\left[\begin{array}{rlll}
-y_{\mathrm{f}}\left(t_{k}\right)-y_{\mathrm{f}}^{(1)}\left(t_{k}\right) \ldots & -y_{\mathrm{f}}^{(n-1)}\left(t_{k}\right) \\
u_{0 \mathrm{f}}\left(t_{k}\right) u_{0 \mathrm{f}}^{(1)}\left(t_{k}\right) \ldots & u_{0 \mathrm{f}}^{(m)}\left(t_{k}\right) & 1
\end{array}\right]
\end{array}
$$

Now, the vector of parameters



can be estimated from the equation

$$
y_{\mathrm{f}}^{(n)}\left(t_{k}\right)=\boldsymbol{\Theta}^{T}\left(t_{k}\right) \boldsymbol{\Phi}\left(t_{k}\right)+\mu\left(t_{k}\right) .
$$

\subsection{Linear part of the controller}

The DLP is inserted into the control loop as shown in Fig. 6. In the scheme, $w$ is the reference signal, $v$ is the disturbance, $y$ is the controlled output, $u_{0}$ is the input to the ELM and $e$ is the tracking error.

The transfer function $G(\mathrm{~s})$ of the ELM is given

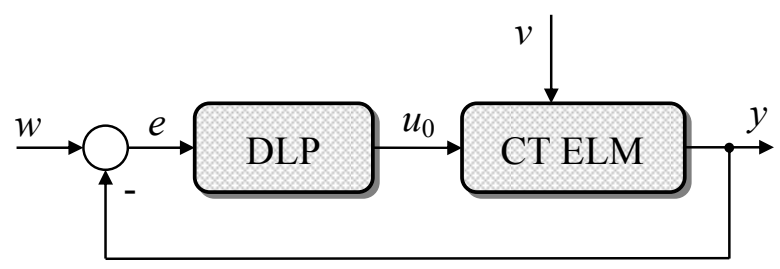

Fig. 6. Simplified scheme of the control loop.

by (14). Both the reference $w$ and the disturbance $v$ are considered to be step functions with transforms

$$
W(s)=\frac{w_{0}}{s}, V(s)=\frac{v_{0}}{s}
$$

The transfer function of the DLP is in the form

$$
Q(s)=\frac{U_{0}(s)}{E(s)}=\frac{q(s)}{p(s)}
$$

where $q$ and $p$ are polynomials in $s$, and, $\operatorname{deg} q \leq \operatorname{deg} p$.

The controller design described in this section stems from the polynomial approach. General conditions required to govern the control system properties are formulated as stability, internal properness, asymptotic tracking of a step reference and step disturbance attenuation.

It is well known from the algebraic control theory that a controller which satisfies above requirements is in the polynomial ring given by a solution of the polynomial (Diophantine) equation

$$
a(s) p(s)+b(s) q(s)=d(s)
$$

with a stable polynomial $d(s)$ on the right side.

For step input signals $w$ and $v$, the polynomial $p$ is in the form

$$
p(s)=s \tilde{p}(s) .
$$

For $\operatorname{deg} a=2$ and step input signals, the degrees of unknown polynomials in (25) and (26) are given as

$$
\operatorname{deg} q=2, \operatorname{deg} \tilde{p}=1, \operatorname{deg} d=4
$$

and, the controller transfer function takes the form

$$
Q(s)=\frac{q(s)}{s \tilde{p}(s)}=\frac{q_{2} s^{2}+q_{1} s+q_{0}}{s\left(s+p_{0}\right)} .
$$

In this paper, the polynomial $d$ with roots determining the closed-loop poles is chosen as

$$
d(s)=n(s)(s+\alpha)^{2}
$$

where $n$ is a stable polynomial obtained by spectral factorization

$$
a^{*}(s) a(s)=n^{*}(s) n(s)
$$

and $\alpha$ is the selectable parameter that can usually be chosen by way of simulation experiments. Note that 
a choice of $d$ in the form (29) provides the control of a good quality for aperiodic controlled processes. The polynomial $n$ has the form

$$
n(s)=s^{2}+n_{1} s+n_{0}
$$

with coefficients

$$
n_{0}=\sqrt{a_{0}^{2}}, n_{1}=\sqrt{a_{1}^{2}+2 n_{0}-2 a_{0}} .
$$

The controller parameters can be obtained from solution of the polynomial equation (25).

Evidently, the controller parameters can be adjusted by the selectable parameter $\alpha$. The complete adaptive control system is shown in Fig. 7.

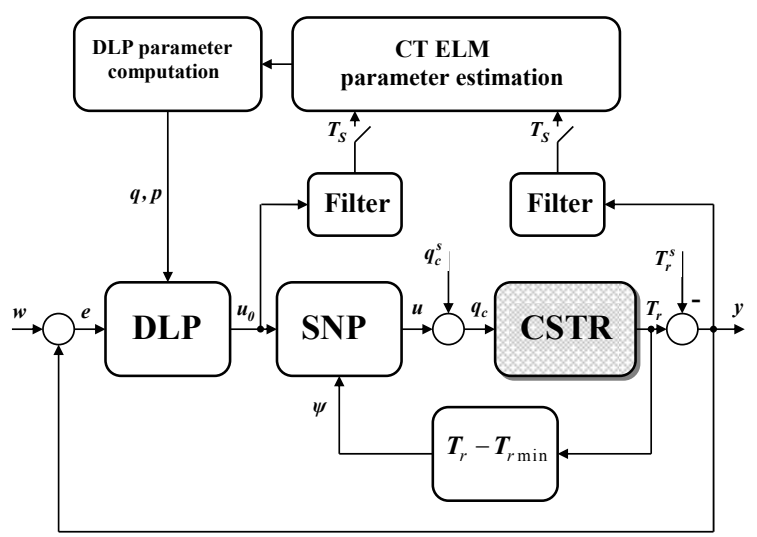

Fig. 7. Adaptive control system.

\section{Control Simulations}

The control simulations were performed in a neighbourhood of the operating point $\left(q_{\mathrm{c}}^{\mathrm{s}}=0.08\right.$ $\mathrm{m}^{3} \min ^{-1}, T_{\mathrm{r}}^{\mathrm{s}}=324.8 \mathrm{~K}$ ). For the start (the adaptation phase), a $\mathrm{P}$ controller with a small gain was used in all simulations.

The effect of the pole $\alpha$ on the control responses is transparent from Figs. 8 and 9. Here, on the basis of precomputed simulations, three values of $\alpha$ were selected. The control results show sensitivity of the controlled output and the input coolant flow rate to $\alpha$. Obviously, careless selection of this parameter can lead to controlled outputs with overshoots or even to instability. Further, an increasing $\alpha$ leads to higher values and changes of the input signals. This fact can be important in control of some reactors where expressive input changes are undesirable.

A presence of the integrating part in the DLP enables rejection of various step disturbances entering into the process. Here, step disturbances $\Delta c_{\text {Af }}= \pm 0.1 \mathrm{kmolm}^{-3}$ at times $t_{\mathrm{v} 1}=210 \mathrm{~min}$ and $t_{\mathrm{v} 2}=510 \mathrm{~min}$ were injected into the CSTR. The DLP parameters were estimated only in the first

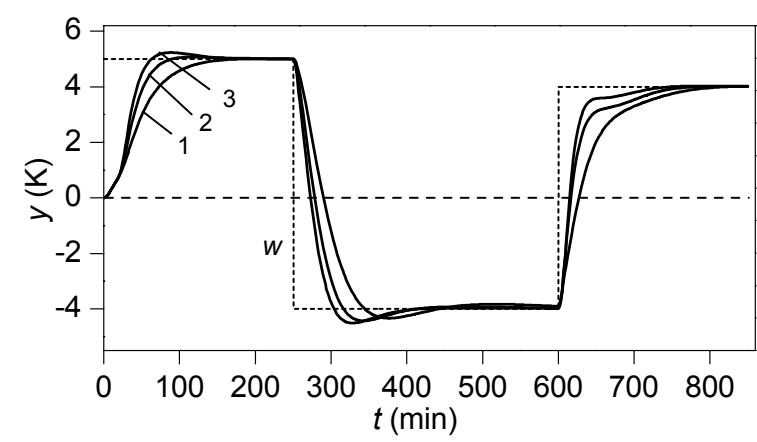

Fig. 8. Controlled output for $\alpha=0.05$ (1), $\alpha=0.075$ (2), $\alpha=0.1$ (3).



Fig. 9. Coolant flow rate for $\alpha=0.05$ (1), $\alpha=0.075$ (2), $\alpha=0.1$ (3).

(tracking) interval $t<200 \mathrm{~min}$. The experiences of authors of this paper proved that an utilization of recursive identification in the phase of a constant reference and in a presence of step disturbances decreases the control quality. From this reason, during interval $t \geq 200 \mathrm{~min}$, fixed DLP parameters were used. The controlled output responses are shown in Fig. 10.

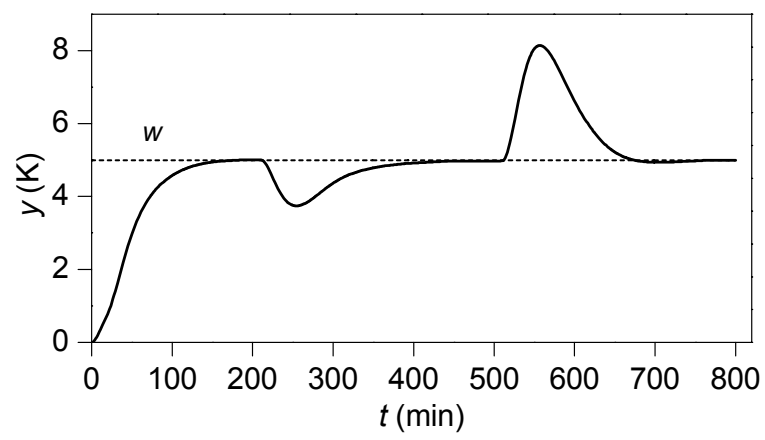

Fig. 10. Step disturbance rejection.

An influence of the SNP is evident from the control responses shown in Fig. 11. Here, the standard adaptive control without the nonlinear part of the controller was used. The simulation has been performed under the same conditions as by above presented cases for $\alpha=0.075$. The responses in Fig. 11 show priority of the nonlinear control especially for greater changes of the reference signal. 




Fig. 11. Comparison of nonlinear adaptive control (1) with standard adaptive control (2).

\section{Conclusions}

In this paper, one approach to the nonlinear continuous-time adaptive co ntrol of the reactant temperature in a continuous stirred tank reactor was proposed. The control strategy is based on a factorization of a controller into the linear and the nonlinear part. A design of the controller nonlinear part employs simulated or measured steady-state characteristics of the process and their additional modifications. Then, the system consisting of the controller nonlinear part and a nonlinear model of the CSTR is approximeted by a continuous time external linear model with parameters obtained through direct recursive parameter estimation. The resulting continuous-time controller linear part is derived using the polynomial approach and given by a solution of a polynomial equation. Tuning of its parameters is possible via closed-loop pole assignment. The presented method has been tested by computer simulation on the nonlinear model of the CSTR with a consecutive exothermic reaction. Simulation results demonstrated an applicability of the presented control strategy and its usefulness especially for greater changes of input signals in strongly nonlinear regions.

\section{Acknowledgment}

The authors wish to thank to the Ministry of Education of the Czech Republic (MSM7088352101) for financial support. This article was created with support of Operational Programme Research and Development for Innovations co-funded by the European Regional Development Fund (ERDF) and national budget of Czech Republic within the framework of the Centre of Polymer Systems project (reg.number: CZ.1.05/2.1.00/03.0111).

References:

[1] A. Astolfi, D. Karagiannis, and R. Ortega, Nonlinear and adaptive control with applications, Springer-Verlag, London, 2008.
[2] Cs. Bányász, and L. Keviczky, A Simple PID regulator applicable for a class of factorable nonlinear plants, in Proc. American Control Conference, Anchorage, Alaska, 2002, pp. 2354-2359.

[3] Chyi-Tsong Chen1, Yao-Chen Chuang1, and Yao-Chen Hwang, A simple nonlinear control strategy for chemical processes, in Proc. 6th Asian Control Conference, Bali, Indonesia, 2006, pp. 64-70.

[4] J.-P. Corriou, Process control. Theory and applications, Springer - Verlag, London, 2004.

[5] P. Dostál, F. Gazdoš, V. Bobál, and J. Vojtěšek, Adaptive control of a continuous stirred tank reactor by two feedback controllers, in Proc. 9th IFAC Workshop Adaptation and Learning in Control and Signal Processing ALCOSP'2007, Saint Petersburg, Russia, 2007, pp. P5-1 - P5-6.

[6] P. Dostál, J. Vojtěšek, and V. Bobál, Simulation of adaptive control of a continuous stirred tank reactor, in Proc. 23rd European Conference on Modelling and Simulation, ECMS 2009, Madrid, Spain, 2009, pp. 625-630.

[7] H. Garnier, and L. Wang (eds.), Identification of continuous-time models from sampled data, Springer - Verlag, London, 2008.

[8] P. Ioannou, and B. Fidan, Adaptive control tutorial, SIAM, Philadelphia, 2006.

[9] V. Kučera, Diophantine equations in control A survey, Automatica, Vol. 29, 1993, pp. 13611375 .

[10] B.A. Ogunnaike, and W.H. Ray, Process dynamics, modeling, and control, Oxford University Press, New York, 1994.

[11] G.P. Rao, and H. Unbehauen, Identification of continuous-time systems, IEE Proc.-Control Theory Appl., Vol. 153, 2006, pp. 185-220.

[12] L.D. Schmidt, The engineering of chemical reactions, Oxford University Press, New York, 2005.

[13] S. Sung, and J. Lee, Modeling and control of Wiener-type processes, Chemical Engineering Science, Vol. 59, 2004, pp. 1515-1521.

[14] H. Vallery, M. Neumaier, and M. Buss, Anticausal identification of Hammerstein models, in Proc. European Control Conference 2009, Budapest, Hungary, 2009, pp. 1071-1076.

[15] T.L. Vincent, and W.J. Grantham, Nonlinear and optimal control systems, John Wiley \& Sons, New York, 1997.

[16] J. Vörös, Recursive identification of Wiener systems with two segment polynomial nonlinearities, Journal of Electrical Engineering, Vol. 59(1), 2008, pp. 40-4 